\title{
Life after personalized adaptive locomotor training: a qualitative follow-up study
}

\author{
Hardeep Singh ${ }^{1,2} \cdot$ Jaya Sam $^{1,3} \cdot$ Mary C. Verrier ${ }^{1,2,3} \cdot$ Heather M. Flett ${ }^{1,3} \cdot$ B. Catharine Craven $\mathbb{1}^{1,2,4} \cdot$ \\ Kristin E. Musselman $\mathbb{1}^{1,2,3}$
}

Received: 6 October 2017 / Revised: 26 December 2017 / Accepted: 27 December 2017

(C) International Spinal Cord Society 2018

\begin{abstract}
Study Design Qualitative follow-up study.

Objectives Intensive locomotor training can improve physical and psychological functioning for individuals with spinal cord injury. Few studies have examined long-term effects of locomotor training. Specifically, there is a lack of qualitative followup that provide insight into participants' perceptions of the effects of locomotor training on level of function and daily life. This study aimed to gain insight into participants' perceptions of intensive locomotor training and whether participation influenced the level of function and community living 1-2 years after training.

Setting Tertiary rehabilitation facility in Ontario, Canada.

Methods Participants were six individuals who had lived with spinal cord injury between 1.9 and 2.7 years at the time of the interviews and had completed locomotor training during the subacute phase of injury. Semi-structured interviews explored participants' daily experiences and level of function after locomotor training. Interviews were analyzed using thematic analysis.

Results Three themes were identified. (1) Outcomes: Personalized Adapted Locomotor Training led to transferable gains from the program to daily functioning and eased transitions out of the rehabilitation hospital. (2) Continuing the rehabilitation journey: following disappointment after training ended, recovery was perceived incomplete regardless of current functional status. Endeavors were now directed to maintaining gains achieved during the program. (3) Challenges: since discharge from Personalized Adapted Locomotor Training, participants identified changes in their psychological well-being and the risk of falls as challenges.

Conclusions Personalized Adapted Locomotor Training was a positive experience. The identified challenges present future opportunities for the improved delivery of intensive locomotor training programs.
\end{abstract}

Electronic supplementary material The online version of this article (https://doi.org/10.1038/s41394-018-0037-z) contains supplementary material, which is available to authorized users.

Kristin E. Musselman

Kristin.Musselman@uhn.ca

1 Toronto Rehabilitation Institute-University Health Network, Toronto, Canada

2 Rehabilitation Sciences Institute, University of Toronto, Toronto, Canada

3 Department of Physical Therapy, University of Toronto, Toronto, Canada

4 Division of Physical Medicine and Rehabilitation, Faculty of Medicine, University of Toronto, Toronto, Canada

\section{Introduction}

Restoring ambulatory function is a high priority for the rehabilitation of individuals with incomplete spinal cord injuries (SCI) [1]. Over the past four decades, researchers have examined the effectiveness of locomotor training to retrain ambulatory function in individuals with incomplete SCI [2-6]. There is growing evidence supporting the physical $[7,8]$, functional, and psychological benefits of locomotor training $[9,10]$.

We previously reported on the immediate post-training benefits of Personalized Adaptive Locomotor Training (PALT) for individuals with subacute, incomplete SCI [10]. PALT is an individualized and intensive locomotor training program that involves body weight-supported treadmill training (BWSTT) and overground walking. Objective 
improvements in overground walking speed, endurance, and balance were seen following PALT [11], and participants reported numerous perceived benefits through interviews conducted immediately upon conclusion of PALT [10].

Few studies have examined the longer-term (i.e., more than 3 months post training) benefits of locomotor training programs [12-14]. These studies have focused on the maintenance of walking ability. However, Hicks and colleagues [13] also reported decreases in subjective wellbeing and satisfaction with physical function, as measured by standardized questionnaires, 8 months following the completion of 12 weeks of BWSTT.

Exploring the experiences of participants 1-2 years after completing locomotor training can provide valuable insight into how training protocols may be improved, and to understand the long-term impact of rehabilitation programs [14]. To-date, no prior qualitative follow-up studies have explored how individuals with SCI are functioning in the community 1-2 years after participation in a locomotor training program, such as PALT. Hence, the purpose of the current study was to gain insight into participants' perceptions of PALT and whether participation in PALT had an influence on their level of function and community living 1-2 years following discharge from PALT.

\section{Methods}

\section{Design}

A qualitative study using an inductive thematic analysis allowed us to describe how individuals with SCI are functioning in daily life after participation in PALT.

\section{Settings and participants}

PALT was completed at the Lyndhurst Centre, Toronto Rehabilitation Institute - University Health Network (TRIUHN), located in Toronto, Canada. In this follow-up study, inclusion required participants to have previously participated in PALT and a semi-structured interview conducted upon completion of training [10], and to be able to participate in a telephone interview lasting $60 \mathrm{~min}$. A purposeful sample [15] of seven participants who previously completed PALT were invited to participate in this study; six of whom agreed. Ethical approval was obtained from the Research Ethics Board of the University Health Network. We certify that all applicable institutional and governmental regulations concerning the ethical use of human volunteers were followed during the course of this research.

\section{PALT intervention}

All participants completed PALT as outpatients. PALT was an individualized and intensive locomotor training regimen, occurring four times per week for a total of 49-131 sessions. PALT consisted of $60 \mathrm{~min}$ of BWSTT and $30 \mathrm{~min}$ of overground training. The overground training may have also incorporated upper extremity training and core strengthening. PALT was tailored to each participant's needs based upon their physical and functional abilities and their goals. Participants were discharged from PALT when their performance on measures of walking and balance plateaued [10].

\section{Data collection}

Semi-structured interviews were focused on the transference of skills gained in PALT to community living. In our previous study, we presented results from semi-structured interviews that were conducted immediately upon completion of PALT. Participants identified a lack of fall prevention training in PALT and a motivation to self-manage their rehabilitation [10]. Hence, questions about falls and current rehabilitation were also incorporated in the interview guide (see supplementary material). Telephone interviews were selected to accommodate participants who lived at a distance (i.e., $>100 \mathrm{~km}$ ) from the Lyndhurst Centre. A researcher with qualitative research experience (H.S.), who was not directly involved in the administration of PALT, conducted the interviews with each lasting $\sim 35-45 \mathrm{~min}$. All interviews were audio-recorded and transcribed verbatim. All participants were assigned pseudonyms.

\section{Data analysis}

Transcribed interviews were transferred to Nvivo 11 (QSR International Pty Ltd., Burlington, MA) [16] for data storage and analysis. Braun and Clarke's [17] inductive approach to thematic analysis was used to analyze the transcribed data. An inductive approach allows the identification of themes that are tied closely to the data [18]. Transcripts were read several times by two authors (H.S. and J.S.). The authors then independently created initial codes and identified overarching themes. The identified themes were compared and discussed until an agreement was established between the authors [17]. The themes were compared to the transcripts by three additional authors (K.E. M., M.V., and H.F.). Through discussions (peer debriefing), the final set of themes was formed [19]. Reflexive notes and a data audit trail were kept by the lead author to enhance the transparency of the research $[20,21]$. To enhance credibility, participants were provided a copy of their transcripts (member-checking) to verify accuracy of transcription [19]. 
Table 1 Participant demographics

\begin{tabular}{|c|c|c|c|c|c|c|c|}
\hline Participant & Sex & Age & MOI & $\begin{array}{l}\text { Time post } \\
\text { injury (years) }\end{array}$ & $\begin{array}{l}\text { Level of } \\
\text { Injury }\end{array}$ & AIS & Current mobility aid \\
\hline Lilly & $\mathrm{F}$ & 49 & NT & 2.6 & $\mathrm{~T} 8$ & $\mathrm{D}$ & $\begin{array}{l}\text { Walking stick } \\
\text { (outdoors) }\end{array}$ \\
\hline Fred & M & 59 & $\mathrm{~T}$ & 2.7 & $\mathrm{C} 5$ & $\mathrm{D}$ & No mobility aid \\
\hline Justin & M & 54 & $\mathrm{~T}$ & 2.3 & $\mathrm{~T} 3$ & $\mathrm{C}$ & Wheelchair \\
\hline Kent & M & 58 & $\mathrm{~T}$ & 2 & $\mathrm{C} 4$ & $\mathrm{D}$ & $\begin{array}{l}\text { Walking poles (indoors) } \\
\text { or walker (outdoors) }\end{array}$ \\
\hline Ann & $\mathrm{F}$ & 65 & NT & 2.1 & $\mathrm{C} 7$ & $\mathrm{D}$ & Wheelchair \\
\hline Ethan & M & 61 & $\mathrm{~T}$ & 1.9 & $\mathrm{C} 4$ & D & No mobility aid \\
\hline Mean Range & & $\begin{array}{l}57.7 \text { (SD } \\
5.6) 49- \\
65\end{array}$ & $\begin{array}{l}2.3(\text { SD 0.3) } \\
1.9-2.7\end{array}$ & & & & \\
\hline
\end{tabular}

MOI method of injury, AIS American Spinal Injury Association Impairment Scale, $T$ traumatic, NT nontraumatic

\section{Results}

Six participants (two females) participated. Four had a traumatic SCI and two had a non-traumatic SCI. Participants were aged 49-65 years and had lived with SCI between 1.9 and 2.7 years at the time of the interviews. Two were employed, one was retired and three were not employed. There was no change in the primary means of mobility for each participant since discharge from PALT (see Table 1).

Three main qualitative themes emerged from the interviews: (1) PALT outcomes, (2) continuing the rehabilitation journey, and (3) challenges experienced since discharge from PALT. Themes, subthemes, and supporting quotes are listed in Table 2.

\section{Theme 1: PALT outcomes}

Regardless of their current functional status, all participants believed that PALT played a vital role in their rehabilitation journey. Participants cited two valuable outcomes from PALT: (1) transferrable gains from the program to community living and (2) facilitation of transitions as participants transitioned from the rehabilitation hospital to living in the community.

\section{Transferrable gains from program}

The training activities and high intensity of PALT contributed to physical and functional improvements that translated into participants' improved functioning in the community. Justin (wheelchair user) explained, "(The program) wasn't only walking...We were stretching and working on my core and that helped me with dressing and moving, in the bed, sitting, and all this stuff." Justin found that PALT also allowed him to become aware of his physical tolerance: "You learn your body again...you are pushing...so you learn your limitations and how much your muscles can take." In gaining this knowledge about his body, this prevented him from overexerting himself and allowed him to engage in activities within his tolerance threshold. Ann, another wheelchair user, added that the improvements she obtained from PALT increased her independence, “If I hadn't come as far as I did on the program I would have been more dependent on my husband and people around me." Ethan (no mobility aid) explained, "you build confidence by being immersed in the program by the repetition, so you gain strength quickly, you gain confidence quickly, just through the progress itself." Engaging in these restorative activities in a supervised setting fostered a sense of independence and self-confidence in the participants. This was seen as crucial to the participants during the rehabilitation process as it empowered them to become more autonomous when attempting to execute day-to-day activities in an unsupervised environment.

\section{Facilitation of transitions}

Several participants explained that attending PALT as an outpatient four times a week eased their transition out of the rehabilitation hospital. PALT allowed the participants the ability to structure and adjust their weekly routines around the program. Fred (no mobility aid) shared his view on the subject, "It did give me structure in my days at a time where getting out of the inpatient and getting into the outpatient, you needed some structure to help you." In addition, through interactions with the clinicians, participants experienced a sense of emotional support as they coped with the severity of their injury. Although ending PALT was a difficult transition for the participants, the program assisted Fred in getting "back onto the road of comparative normalcy" as he settled back into pre-injury environments 


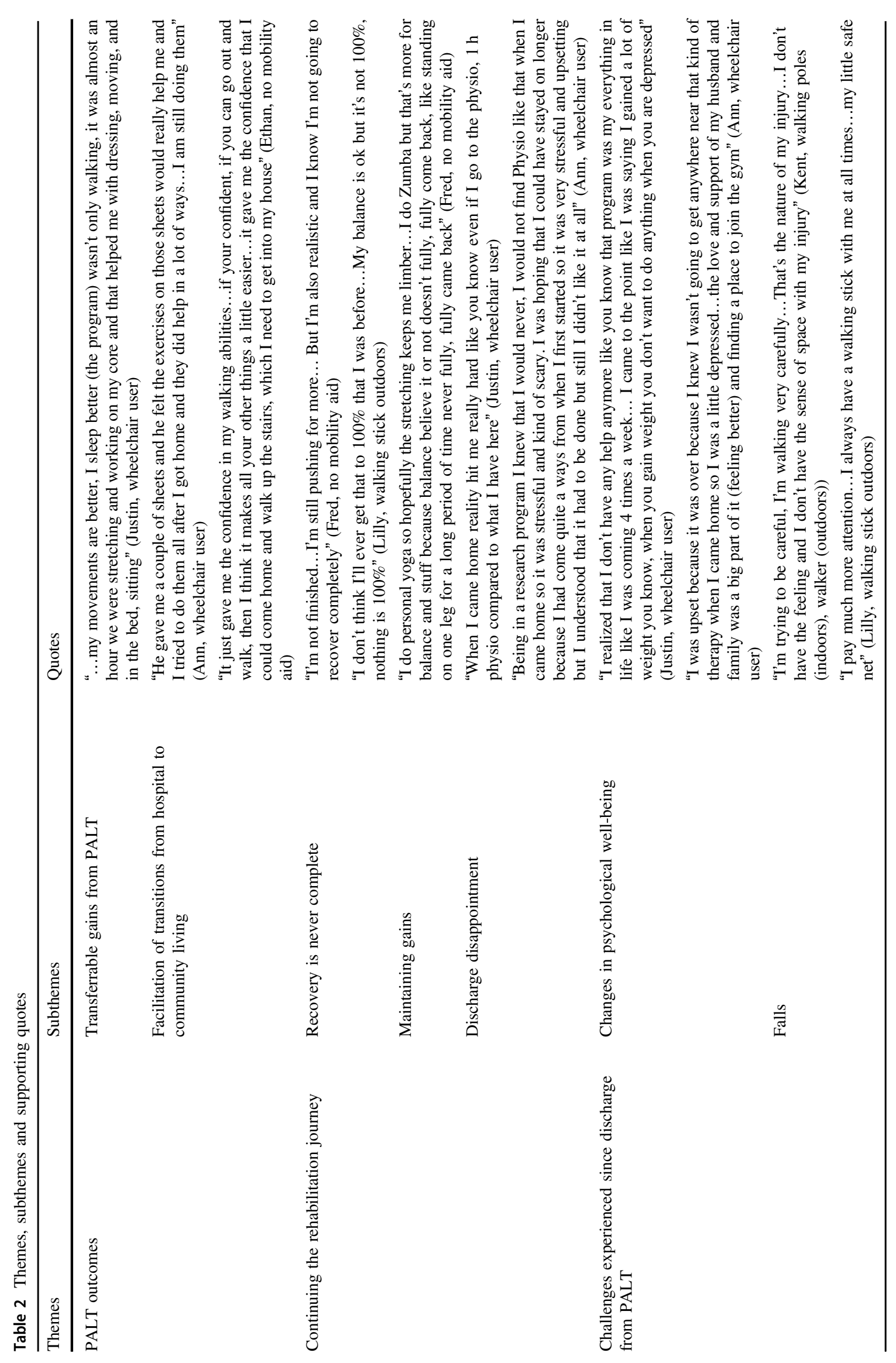


and routines. Others found that the resources provided by PALT clinicians, such as home exercise programs and community resource pamphlets, guided them in selecting the appropriate facilities to continue their community rehabilitation.

\section{Theme 2: continuing the rehabilitation journey}

Life after PALT marked a new and more stable phase in the participants' rehabilitation journey. With limited or no professional guidance, responsibility to manage rehabilitation activities was solely on the participant and her/his family. Although discharge was expected, participants revealed experiencing a period of disappointment after PALT ended. They endorsed beliefs that their recovery would never be complete and their gains from PALT must be maintained through regular engagement in physical activity.

\section{Discharge disappointment}

Transitioning from a highly structured program to no longer having professional rehabilitation guidance was an onerous experience for participants. After being discharged from PALT, most participants experienced a period of disappointment because of the loss of guidance and intensive rehabilitation provided by PALT. Ann (wheelchair user) recalled after her discharge: "It was almost like when you leave home when you're a child on your own, it's like you lost your safety net. I was very disappointed..."

Forming new routines and activities to occupy their weekly schedules was challenging, especially for those who did not return to work by the time PALT ended. Disappointment also stemmed from being unable to find comparably competent community rehabilitation programs. More specifically, Justin (wheelchair user) explained:

Right after it ended I was pumped, I wanted to walk. I was going to walk myself but after a while you realize you need still a professional somebody who will...tell you are doing it right or you were doing it wrong so that was missing... I can walk on a walker and I feel happy with that but when I came home reality hit me really hard...

Eventually creating a regular routine and engaging in community physical activity programs to maintain gains diminished their disappointments. Interestingly, this feeling of disappointment was not shared by one participant. In fact, Lilly (walking stick outdoors) felt ready for her discharge; this participant had reached a high level of functioning having met and exceeded most of her rehabilitation goals.

\section{Recovery is never complete}

There was a consensus that recovery from an SCI was incomplete regardless of a participant's functional status. Although many participants noted significant physical improvements while completing PALT, they felt their recovery was still not finished. Many still strived for and expected further improvements. Higher functioning individuals focused on refining their skills such as balance, muscle mass, fine motor skills, range of motion, and activity tolerance, while individuals with lower levels of bodily function felt, "it's still a long way to go" (Justin, wheelchair user). Overall, there was an agreement that the recovery process was ongoing and, although PALT had provided an adequate starting point for their rehabilitation, the participants indicated that they would not be satisfied until they regained their pre-injury level of function.

\section{Maintaining gains}

Participants expressed concerns about maintaining their functional and physical gains. Physical improvements were understood as being temporary and that they must be maintained with continued participation in physical activity: "(Exercise) is a daily routine that I have to do forever... If you don't use the muscles, don't bend and stretch, and that kind of thing, you freeze up and nothing will work" (Ann, wheelchair user). Moreover, there was a general consensus that self-directed recreational programs such as working out in a regular gym or engaging in exercises at home was more financially feasible than long-term participation in an intensive community rehabilitation program. Participating in physical activity programs was thought to be sufficient in aiding participants in maintaining their gains or "to keep the nervous system entertained" (Fred, no mobility aid).

\section{Theme 3: challenges experienced since discharge from PALT}

Over the 1-2 year period after completing PALT, participants described their current physical limitations as well as their varying tolerance levels. Following PALT, three participants described a difficult adjustment to their new identities and functional abilities, while three others stated that they were able to adjust more easily. Two common challenges participants confronted during this pivotal period were decline in psychological well-being after discharge from PALT and the risk of injury caused by falls.

\section{Psychological well-being}

While participating in PALT, participants described being motivated and hopeful that they would regain prior levels of 
function or, at least something close to it. However, those who perceived their abilities were furthest from their preinjury levels experienced greater decline in their psychological well-being. In particular, Justin (wheelchair user) explained, "After that program finished, when I came home in December, it was obviously the holidays and after that I got really depressed." He further explained that comparing current to prior level of function before sustaining an SCI, and gaining weight since discharge from PALT, caused him to become depressed and admitted to contemplating suicide. When asked whether he had informed a health professional, he explained "I didn't want to bother them." After a few months, his depression became worse and he eventually consulted his family physician. Justin also made a commitment to join a community gym.

Some spoke about their current functional abilities in comparison to their pre-injury "normal" abilities and how they lost their sense of self as a result. After PALT ended, Ethan (no mobility aid) described feeling upset because he believed he could no longer participate in meaningful activities: "working, motorcycle riding, woodworking, hockey, golf, everything that I did before was taken away from me." Since then, Ethan has resumed participation in some of those activities. Kent (ambulatory with walking poles (indoors) or walker (outdoors) indicated "zero" satisfaction with his current level of function and explained, "I am not the same person anymore...Y You see the life...in the community, so you compare everything. So [the depression] gets worse and worse." He sought medical intervention 4 months after experiencing depressive symptoms. Ann, a wheelchair user, experienced a brief period of psychological decline immediately after PALT ended. Nevertheless, she came to terms with her current level of function, "Now I am fine. I deal with things as they come. I have learned to be very patient because things can't happen very fast anymore and I am in a good place right now." All but Kent described eventually coming to terms with their current reality and moving on with their lives. While experiencing these periods of low psychological well-being, participants reported concurrent declines in physical activity, which was later ameliorated following improvements in their mental health.

\section{Falls}

The risk of falling was an issue the participants encountered on a daily basis. Four of the six participants had experienced at least one fall since being discharged from PALT, and three participants experienced "countless" near falls. Lilly, an ambulatory participant, reported a fall that resulted in a wrist fracture. Concerns about sustaining injuries as a consequence of falls varied with some participants being fearful of falling, whereas others accepted falls as an everyday risk. In contrast, others who experienced less injurious falls preferred the terms "concern" and "apprehension" rather than an outright "fear" of falls. Fred (no mobility aid) clarified, "Fear would keep me from doing something. Apprehension would make me much more focused when I was doing an activity in the future."

Most participants took responsibility for falls and blamed "intrinsic/internal factors" such as fatigue, lack of attention, and their body's inability to support their body weight. Participants appeared to have developed fall prevention strategies to avoid future falls based on their prior experiences. For example, although Lilly did not rely on a mobility aid for ambulation, she kept a walking stick with her for outdoor ambulation as a "safety net". Many participants indicated paying more attention to their body movements and the external environment.

\section{Discussion}

The current study examined the experiences of participants 1-2 years post-discharge from PALT. Qualitative perspectives after locomotor training have not been studied extensively. The findings of this study offer new insight into the experiences of participants after their discharge from an intensive locomotor training program completed during the subacute phase of injury. The results of this study uncovered the positive role PALT can play in the rehabilitation journey following a life-altering SCI. In particular, PALT facilitated transitions from hospital to community by providing structure in the participants' routines as well as a social environment that fostered hope for their futures through establishing meaningful rehabilitation goals. The end of PALT was marked by a period of disappointment after which participants focused their efforts on maintaining rehabilitation gains all the while believing that their recovery may never be "complete". Nonetheless, challenges such as psychological decline and falls were prevalent after discharge from PALT. These described challenges offer future opportunities for PALT clinicians to provide targeted interventions.

A new insight revealed from this research was the difficulties the participants faced after discharge from PALT. With the intensive and individualized locomotor training and therapeutic exercises, not only did PALT play a vital role in improving participants' performance from a physical standpoint, it also provided structure to their routines and a supportive social environment. Although prior research has revealed hypo-activity after discharge from rehabilitation and challenges in rediscovering new situations [22-24], decreased activity had not been previously recognized as a discharge concern for locomotor training. During this follow-up period after completion of PALT, participants faced emotional and psychological difficulty with the 
sudden removal of the professional guidance offered by PALT clinicians.

Our findings indicate that the experiences of participants since discharge from PALT varied based on their current levels of satisfaction with their physical abilities. Since their discharge, all participants, except Kent, noted improvements in their psychological well-being a few months following a period of decline. These results suggest that there is an adjustment period after discharge from PALT. Although most participants adjusted well to life after PALT, some noted difficulty with the adjustment. While some required medical intervention, others attributed their improvements to re-engaging in regular physical activity and their social support systems. Physical activity and social support were previously found to have health and psychological benefits in individuals living with SCI [25, 26]. It is important to recognize that two participants were hesitant in sharing depressive symptoms with health-care professionals. Given the varied experiences of participants, it is recommended that PALT should include routine follow-ups to identify those who may be experiencing a decline in their psychological well-being. These findings also support the importance of rehabilitation programs in directing greater efforts toward fostering a socially supportive network in the community. It suggests a need for less abrupt discharges and increased guidance in establishing meaningful routines prior to an individual being discharged from PALT entirely $[27,28]$. In agreement with prior research, intensive training such as PALT can be followed by a less intensive intervention in conjunction with counseling [22, 28, 29]. This may be a more supportive method to discharge individuals from PALT and address potential decline in psychological health experienced by some participants after discharge.

Even though PALT helped to empower people to selfmanage their rehabilitation [10], this follow-up study revealed several obstacles individuals with SCI are confronted with while continuing therapy in the community. In spite of a recognized need for continued therapy to maintain physical gains, barriers (i.e., financial and therapy burnout) limited continued participation in community rehabilitation. This finding implies that PALT should shift its focus from encouraging participants to continue their training in the community to educating them; for example, providing individuals with self-management strategies to allow participants to continue their physical training with activities that can be performed at public gyms or in their homes. In this context, self-management of their physical health can allow individuals to continue their rehabilitation in a sustainable manner.

Falls and fear of falls are a concern for individuals with SCI [30-32]. Similar to results from Jorgensen and Roaldsen [32], the current research has found that individuals with SCI accept falls as an everyday risk. As acknowledged by prior research [30, 33, 34], participants in this study also attributed falls to be a result of personal shortcomings and intrinsic/internal factors. Consistent with our previous findings [10], PALT helped participants gain an awareness of their own physical abilities and tolerances. In effect, this equipped participants with the necessary knowledge to develop their own strategies to avoid falls outside the confines of the clinic. In support of our previous study [10], the current study supports the need for PALT to incorporate training on how to prevent falls into the program.

Several limitations in this study are worth considering. First, the small sample limits generalizations [35]. Further, limitations of the telephone interviews include reduced depth of discussions and an absence of visual/non-verbal communication cues $[36,37]$. Recall bias may be a concern with some interview questions that asked participants to recall their experiences since leaving the program [38]. Finally, the broad range of topics covered during interviews may have limited the depth of the responses. Despite these limitations, this study offers valuable insight into the experiences of participants after they had completed PALT. Future research should continue to explore the perspectives of participants' to improve rehabilitation programs. Findings from this study can be used to help to address the various challenges individuals with SCI face in their daily lives after completing rehabilitation programs.

Acknowledgements This work was supported by the Ontario Neurotrauma Foundation (grant number 2013-SCI-RECOV2-974).

Author contributions All authors contributed to the design, analysis, and writing of this manuscript.

\section{Compliance with ethical standards}

Conflict of interest The authors declare that they have no conflict of interest.

\section{References}

1. Ditunno PL, Patrick M, Stineman M, Ditunno JF. Who wants to walk? Preferences for recovery after SCI: a longitudinal and crosssectional study. Spinal Cord. 2008;46:500-6.

2. Barbeau H, Wainberg M, Finch L. Description and application of a system for locomotor rehabilitation. Med Biol Eng Comput. 1987;25:341-4.

3. Stewart BG, Tarnopolsky MA, Hicks AL, McCartney N, Mahoney DJ, Staron RS, et al. Treadmill training-induced adaptations in muscle phenotype in persons with incomplete spinal cord injury. Mus Nerve. 2004;30:61-8.

4. Behrman AL, Harkema SJ. Locomotor training after human spinal cord injury: a series of case studies. Phys Ther. 2000;80:688-700.

5. Field-Fote EC. Combined use of body weight support, functional electric stimulation, and treadmill training to improve walking ability in individuals with chronic incomplete spinal cord injury. Arch Phys Med Rehabil. 2001;82:818-24. 
6. Behrman AL, Lawless-Dixon AR, Davis SB, Bowden MG, Nair $\mathrm{P}$, Phadke $\mathrm{C}$, et al. Locomotor training progression and outcomes after incomplete spinal cord injury. Phys Ther. 2005;85:1356-71.

7. Buehner JJ, Forrest GF, Schmidt-Read M, White S, Tansey K, Basso DM. Relationship between ASIA examination and functional outcomes in the NeuroRecovery Network locomotor training program. Arch Phys Med Rehabil. 2012;93:1530-40.

8. Harkema SJ, Schmidt-Read M, Behrman AL, Bratta A, Sisto SA, Edgerton VR. Establishing the NeuroRecovery Network: multisite rehabilitation centers that provide activity-based therapies and assessments for neurologic disorders. Arch Phys Med Rehabil. 2012;93:1498-507.

9. Browden MG, Hannold EM, Nair PM, Fuller LB, Behrman AL. Beyond gait speed: a case report of a multidimensional approach to locomotor rehabilitation outcomes in incomplete spinal cord injury. Neurol Phys Ther. 2008;32:129-38.

10. Singh H, Shah M, Flett, HM, Craven BC, Verrier MC, Musselman KE. Perspectives of individuals with sub-acute spinal cord injury after personalized adapted locomotor training. Disabil Rehabil. 2017; http://doi.org/10.1080/09638288.2016.127739.

11. Chan K, Verrier M, Craven BC, Alappat C, Flett H, Furlan JC, et al. Effects of Personalized Adapted Locomotor Training (PALT) on walking function in individuals in the subacute stage after spinal cord injury: a prospective case series. Abstract 7th National Spinal Cord Injury Conference. J Spinal Cord Med. 2017;40:813-69.

12. Musselman KE, Fouad K, Misiaszek JE, Yang YF. Training of walking skills overground and on the treadmill: case series on individuals with incomplete spinal cord injury. Phys Ther. 2009;89:601-11.

13. Hicks AL, Adams MM, Martin Ginis K, Giangregorio L, Latimer A, Phillips SM, et al. Long-term body-weight-supported treadmill training and subsequent follow-up in persons with chronic SCI: effects on functional walking ability and measures of subjective well-being. Spinal Cord. 2005;43:291-8.

14. Wernig A, Nanassy A, Muller S. Maintenance of locomotor abilities following Laufband (treadmill) therapy in para- and tetraplegic persons: follow-up studies. Spinal Cord. 1998;36:744-9.

15. Creswell JW. Qualitative inquiry and research design: choosing among five approaches. 3rd ed. Thousand Oaks, CA: Sage Publishing Ltd.; 2013.

16. QSR International. http://www.qsrinternational.com/product. Accessed 2 May 2017.

17. Braun V, Clarke V. Using thematic analysis in psychology. Qual Res Psychol. 2006;3:77-101.

18. Patton MQ. Qualitative evaluation and research methods. 4th ed. Thousand Oaks, CA: Sage Publishing Ltd.; 1990.

19. Lincoln YS, Guba EG. Naturalistic inquiry. Newbury Park, CA: Sage Publishing Ltd.; 1985.

20. Finlay L. Negotiating the swamp: the opportunity and challenge of reflexivity in research practice. Qual Res. 2002;2:209-30.

21. Carcary M. The research audit trial - enhancing trustworthiness in qualitative inquiry. EJBRM. 2009;7:11-24.

22. Van den Berg-Emons HJG, Bussmann JBJ, Haisma JA, Sluis TA, van der Woude LH, Bergen MP, et al. A prospective study on physical activity levels after spinal cord injury during inpatient rehabilitation and the year after discharge. Arch Phys Med Rehabil. 2008;89:2029-101.

23. Levins SM, Redenbach DM, Dyck I. Individual and societal influences on participation in physical activity following spinal cord injury: a qualitative study. Phys Ther. 2004;84:496-509.

24. Jannings W, Pryor J. The experiences and needs of persons with spinal cord injury who can walk. Disabil Rehabil. 2012;34:1820-6.

25. Williams TL, Smith B, Papathomas A. The barriers, benefits and facilitators of leisure time physical activity among people with spinal cord injury: a meta-synthesis of qualitative findings. Health Psychol Rev. 2014;8:404-25.

26. Rintala D, Young M, Hart K, Clearman R, Fuhrer M. Social support and the well-being of persons with spinal cord injury living in the community. Rehabil Psychol. 1992;37:155-63.

27. Dickson AR, Ward R, O'Brien G, Allan D, O'Carroll R. Difficulties adjusting to post-discharge life following a spinal cord injury: an interpretative phenomenological analysis. Psychol Health Med. 2011;16:463-74.

28. Vissers M, van den Berg-Emons R, Sluis T, Bergen M, Stam H, Bussmann H. Barriers to and facilitators of everyday physical activity in persons with a spinal cord injury after discharge from the rehabilitation centre. $\mathrm{J}$ Rehabil Med. 2008;40:461-7.

29. Pelletier CA, Latimer-Cheung AE, Warburton DE, Hicks AL. Direct referral and physical activity counselling upon discharge from spinal cord injury rehabilitation. Spinal Cord. 2014;52:392-5.

30. Brotherton SS, Krause JS, Nietert PJ. Falls in individuals with incomplete spinal cord injury. Spinal Cord. 2007;45:37-40.

31. Musselman KE, Arnold C, Pujol C, Lynd K, Oosman S. Falls after spinal cord injury: an exploratory study using photovoice. Poster presented at The 55th ISCOS Annual Scientific Meeting; 15 September 2016; Vienna, Austria.

32. Jorgensen V, Roaldsen KS. Negotiating identity and self-image perceptions of falls in ambulatory individuals with spinal cord injury - a qualitative study. Clin Rehabil. 2017;31:544-54.

33. Amatachaya S, Pramodhyakul W, Wattanapan P, Wattanapan P, Eungpinichpong W. Ability of obstacle crossing is not associated with falls in independent ambulatory patients with spinal cord injury. Spinal Cord. 2015;53:598-603.

34. Saunders LL, DiPiro ND, Krause JS, Brotherton S, Kraft S. Risk of fall-related injuries among ambulatory participants with spinal cord injury. Top Spinal Cord Inj Rehabil. 2013;19:259-66.

35. Kukull WA, Ganguli M. Generalizability: the trees, the forest, and the low-hanging fruit. Neurology. 2012;78:1886-91.

36. Creswell JW. Qualitative inquiry and research design: choosing among five traditions. Thousand Oaks, CA: Sage Publications Ltd; 1998.

37. Garbett R, McCormack B. The experience of practice development: an exploratory telephone interview study. J Clin Nurs. 2001;10:94-102.

38. Hassan E. Recall Bias can be a threat to retrospective and prospective research designs. Internet J Epi. 2005;3:1-7. 